\title{
BMJ Open Oral propranolol for treatment of the subgroups of essential tremor: a systematic review and meta- analysis protocol
}

Manyu Zhang (D) , Wei Li, Lan Hu, Li Chen, Liu Yang, Tian Zhang, Hui Shen (D) , Yanan Peng, Shijun Gao, Zhibin Chen, Tan Wang, Zhenqiang Zhao

To cite: Zhang M, Li W, Hu L, et al. Oral propranolol for treatment of the subgroups of essential tremor: a systematic review and metaanalysis protocol. BMJ Open 2020;10:e032096. doi:10.1136/ bmjopen-2019-032096

- Prepublication history and additional material for this paper are available online. To view these files, please visit the journal online (http://dx.doi. org/10.1136/bmjopen-2019032096).

MZ and WL contributed equally.

Received 19 June 2019 Revised 21 November 2019 Accepted 04 December 2019

Check for updates

(c) Author(s) (or their employer(s)) 2020. Re-use permitted under CC BY-NC. No commercial re-use. See rights and permissions. Published by BMJ.

Department of Neurology, First Affiliated Hospital, Hainan Medical University, Haikou, Hainan, China

Correspondence to Dr Zhengiang Zhao; zhenqiang.zhao@qq.com

\section{ABSTRACT}

Introduction Essential tremor (ET), a tremor disorder, is one of the most common movement disorders. Only oral drugs (propranolol, primidone, topiramate, etc ) are still the first-line treatment recommended by the Food and Drug Administration. Propranolol is thought to potentially reduce upper limb action tremor. However, it has a poor effect on axial tremor symptoms, such as essential head tremor and voice tremor. Studies have shown that tremor severity develops over time, possibly producing other clinical tremors and neurological soft signs (such as memory loss, gait abnormalities, balance disorders, etc), which further increases the difficulty of treating tremors. However, some recent studies provide emerging evidence for oral propranolol on subgroups of ET, which is based on the anatomical distribution of ET (lower extremities, head, sound, tongue, etc). This systematic review aims to synthesise these new data to improve the efficacy of propranolol in ET subgroups.

Methods and analysis We will search for randomised controlled trials from the PubMed, MEDLINE, EMBASE, Cochrane Library, UptoDate and PEDro databases from inception to June 2019. All data will be extracted independently by two reviewers and compared at the end of the review. The two reviewers will screen the study quality, and the Cochrane Collaboration's tool in Review Manager (RevMan) V.5.3.3 will be used to evaluate risk of bias. Our primary outcome will be the functional disability component related to tremors, as measured by the FahnTolosa-Marin Tremor Rating Scale subscales B and C. Secondary outcomes will include severity of tremors and quality of life. Narrative and meta-analytical syntheses are planned.

Ethics and dissemination Published aggregated data will be used in this review analysis and therefore no ethical approval is required. The results will be published in peerreviewed journals, and proliferation activities will include diverse social stakeholders, non-academic groups and patients.

PROSPERO registration number CRD42018112580

\section{INTRODUCTION}

Essential tremor (ET) is a chronic, progressive movement disorder occurring in adults, with a prevalence ranging from $0.4 \%$ of the
Strengths and limitations of this study

- A continuously updated data extraction form will be used to ensure data integrity and relevance.

- We will resolve disagreements during the assessment through consensus or discussions with a third investigator.

- Although we will include research published in any language, translation difficulties may occur, which will cause these studies to be excluded.

- Differences in patients, interventions and primary outcomes may mean that meta-analysis cannot be conducted, and narrative and meta-analytical syntheses are planned.

- Multiple limitations may increase the heterogeneity of the study, thereby hampering the results of the meta-analysis.

general population to $5 \%$ of the population over the age of 65 years. ${ }^{1-3}$ It appears that the prevalence of ET increases exponentially in the ageing population. ${ }^{4}$ While the direct cause of ET remains unknown, recent reports have indicated that loss or dysfunction of Purkinje neurons in the cerebellum likely plays a key role in the aetiology of ET, ${ }^{5-8}$ and electrophysiological methods reveal abnormal oscillations in the cortical-ponsalcerebellar-thalamic-cortical loop. ${ }^{9}$ It is still unclear why this network is involved in tremor; however, it is thought to be associated with abnormalities in gamma aminobutyric acid transmission in brain tissues of patients with ET. ${ }^{11}$

Traditionally, ET is defined as bilateral but systematic kinetic and postural tremors of the upper limbs, voice, head, face, chin, legs or a combination of these symptoms. ${ }^{12}$ The incidence of ET usually involves the upper limbs (95\% of patients) according to anatomical distribution; less commonly affected are the lower limbs $(30 \%)$, head 
(25 34\%), sound (12 15\%), tongue $(7 \%)$ face $(5 \%)$ and trunk $(5 \%)$, as described in several previous reports. ${ }^{13-15}$ There are few patients with ET with isolated head tremor. ${ }^{16}{ }^{17}$ Recently, some studies indicated that some complementary neurological signs (ie, other than action tremor), such as mild impaired memory, impaired tandem gait and subtle dystonic body posturing, are present in patients with ET. These clinical symptoms and signs were so mild that they did not suffice for other neurological diagnoses. It was recently proposed that these presentations might be classified as 'essential tremor plus' by the ${ }^{18}$ International Parkinson and Movement Disorder Society in 2018. ${ }^{18}$

Despite the presentation of new viewpoints on ET pathogenesis, the treatment of ET remains merely symptomatic. The therapeutic approach to ET still primarily depends on drugs, although surgery may be an option for patients with refractory ET. As of 2018, propranolol and primidone are still two first-line medications for the treatment of primary tremor, according to the recommendations of the US Food and Drug Administration (FDA) and the European Medicines Agency. ${ }^{18}{ }^{19}$ In particular, the US FDA approved only propranolol for ET. Conventional wisdom is propranolol is only effective against the upper limb action tremor, while axial tremor symptoms, such as essential head tremor and voice tremor, usually respond poorly to propranolol treatments. ${ }^{320}$ Studies have shown that the severity of tremors develops over time, possibly producing other clinical tremors (such as voice tremors) and neurological soft signs (such as memory loss, gait abnormalities, balance disorders, etc), which makes it even more difficult. ${ }^{21} 22$ Therefore, we performed subgroup classification based on anatomical distribution and combined it with soft signs of the nervous system to find the best choice for drug control of primary tremors. However, some recent studies provide emerging evidence for oral propranolol on subgroups of ET, which is based on the anatomical distribution of ET (lower extremities, head, sound, tongue, etc) ${ }^{23-27}$ Integration of these new data is necessary to refine the treatment for the efficacy of propranolol in the subgroups of ET.

The systematic review protocol is performed under the Preferred Reporting Items for Systematic review and Meta-Analysis Protocols (PRISMA-P) guidelines.

\section{Patient and public participation}

No patients will be involved.

\section{Research objective}

The purpose of the systematic review and meta-analysis will be to address the safety and efficacy of propranolol in treating subgroups of ET. The issues of interest to this review are listed below: How effective is the therapy? What is the optimal dosage of the therapy in clinical studies? What are the adverse effects of the therapy? and others.

\section{METHODS}

\section{Eligibility criteria}

Population included

This study will include adult men and women over the age of 16 years with ET diagnosed according to the criteria set by the Tremor Investigation Group, ${ }^{28}$ and the Consensus Statement of the Movement Disorder Society on Tremor. ${ }^{182}$ Individuals with Parkinson's disease, metabolic tremor, drug tremors, toxicity-related tremor, tonic tremor, neurological tremor and functional tremor will be excluded. ${ }^{3}$

\section{Intervention}

The reference intervention is oral propranolol, both long-acting and short-acting formulations. We hypothesise that oral propranolol treatment will be better than other intervention treatments.

\section{Comparators/control}

There are many alternative treatment options for ET, including: (1) Non-operative care with primidone, topiramate, botulinum toxin injections or other drug therapies. (2) Operative care with deep brain stimulation or thalamotomy or gamma knife thalamotomy. ${ }^{30}$ Briefly, the comparator group will include all other treatments.

\section{Outcomes}

\section{Primary and secondary outcomes}

Our primary outcome will be the functional disability component related to tremors, which is measured by the Fahn-Tolosa-Marin Tremor Rating Scale (TRS) subscales $\mathrm{B}$ and C. ${ }^{31}$ Secondary outcomes of interest will include the severity of tremors and quality of life (QoL). Specifically, the severity of tremors will be measured using the Fahn-Tolosa-Marin TRS subscale A and total score, Patient Global Impression (PGI) and Clinical Global Impression (CGI). At the same time, we will use a validated QoL scale or questionnaire to measure QoL, such as the 36-item Short Form or EuroQol. ${ }^{31}$

\section{Study design}

The systematic review protocol will be performed under the PRISMA-P guidelines ${ }^{32}$ and will be reported in accordance with the PRISMA extension statement in the metaanalysis. The completed PRISMA-P checklist for the current review is provided with online supplementary file 1 . We will include all randomised controlled trials (RCTs) that examine the efficacy and safety of propranolol for the treatment of ET. We will not limit language in order to identify all relevant RCTs, both published and unpublished. To improve the internal validity of the review, the Grades in Recommendation, Assessment, Development and Evaluation approach will be used to evaluate RCTs. RCTs have the highest level of evidence for this method. To express our research criteria in a more visual manner, the following table (table 1) has been provided. 
Table 1 The list of inclusion and exclusion criteria

\begin{tabular}{lll}
\hline & Inclusion & Exclusion \\
\hline Population & Adults over the age of 16 years & Parkinsonian tremor \\
& Individuals with diagnosed ET & Metabolic tremor \\
& Toxicity-related tremors & Dystonic tremor \\
& Neuropathic tremor & Functional tremor
\end{tabular}

$\begin{array}{lll}\text { Intervention } & \text { Oral propranolol } \\ \text { Comparators } & \text { Long-acting or short-acting formulation } & \text { Non-operative care with primidone, topiramate, botulinum toxin } \\ & \text { injections or other drug therapies } \\ & \text { Operative care with deep brain stimulation, thalamotomy or gamma } \\ & \text { knife thalamotomy, or the comparator group includes all other } \\ & \text { treatments } \\ & \text { Primary } \\ & \text { Sahn-Tolosa-Marin Tremor Rating Scale } \\ \text { Secondary } & \text { Tremor severity } \\ & \text { Quality of life } & \text { N/A } \\ \text { Stcomes } & \text { Randomised controlled trials }\end{array}$

ET, essential tremor.

\section{The planned start and end dates}

The start date of the study is April 2019, and the end date is February 2020.

\section{Patient and public participation}

No patients will be involved.

\section{Search strategy}

We will perform a comprehensive electronic search of the medical and rehabilitation literature using medical subject headings and text related to ET and propranolol. A comprehensive electronic search of the following databases will be performed, including PubMed, MEDLINE, EMBASE, the Cochrane Library, UptoDate and PEDro, from the beginning of each database to June 2019. Two different experts have developed the search strategy based on the Peer Review of Electronic Search Strategies framework. ${ }^{33}$ Professionals will be asked to review the strategy if necessary. There is an example for the search strategy using the Medline search (online supplementary file 2) that will be modified according to the indexing systems of the other databases. Grey documents that meet the inclusion criteria will also be searched, including papers that have been published, reports on relevant agencies, and unpublished data and manuscripts provided by the original authors.

\section{Study records}

All standards-compliant articles identified in the databases will be imported to EndNote X7 for management. All retrieved articles will be strictly screened by two independent reviewers according to the inclusion criteria.
Simultaneously, for some uncertain articles, the reviewers will browse the title and abstract to determine eligibility. The full texts of each potentially relevant article will strictly adhere to the selection criteria during the title and abstract screening phase. The full texts of all potential articles that meet the inclusion criteria will be obtained and reviewed again. Two independent reviewers will screen the full texts for inclusion. If there is any opposition, we will consult or seek the opinion of the third examiner. The reviewers will have no preferences for authors or journals when screening articles.

\section{Data extraction}

To extract the most relevant data from the article, a predesigned data extraction form will be constructed and will continue to be updated to ensure data integrity and relevance. All data will be independently extracted by the two reviewers and then compared at the end of the review. Divergences will be resolved by consultation or discussion with the third examiner. Reviewers will be impartial in extracting the data. The extracted data form will mainly include the following information: the general information on the publication (author, year), the characteristics of the participants (sex, age, age range, health status), study design, sample size; length of follow-up; the study methods used; the control group; the forms of tremor; the dose of propranolol; the dose of other drugs; the statistical analysis methods; and the effect of the intervention. Only one article will be kept when there are two or more articles derived from the same data. To ensure the integrity and authenticity of the data, the researchers will 
contact the author by email or telephone to request the original data when data are found to be missing. If there are no responses after two consecutive emails and calls, the data will not be included.

\section{Outcomes and prioritisation}

Primary outcome

Our primary outcome will be the functional disability component related to tremors, which is measured by the Fahn-Tolosa-Marin TRS subscales B and C. Rest, posture and tremor are the three elements of TRS, and ETs will be scored using three subscales to assess the severity of the tremor. The three subscales are the posture and the magnitude of the tremor, the ability to perform certain actions and disabilities in daily living due to tremor. Each subscale ranges from 0 to 4 , which represents none, mild, moderate and severe, and the overall maximum scores are 16, 36 and 32 in each subscale. Finally, the scores of the three subscales will be summed to obtain the overall TRS score.

\section{Secondary outcomes}

PGI, CGI and Fahn-Tolosa-Marin TRS subscale A and total score are used to assess the severity of ET. PGI is a scale for patients to self-rate the severity. CGI is a scale for clinicians to assess the severity. At the same time, we will use a validated QoL scale or questionnaire to measure QoL, such as the 36-item Short Form or EuroQol.

\section{Risk of bias and meta-bias}

Two reviewers will separately assess the risk of bias and reporting quality of all included studies. Moreover, each RCT will be assessed in Review Manager (RevMan) V.5.3.3. Each included study will be evaluated using a bias risk table that includes seven items, including random sequence generation, allocation concealment, blinding of participants and personnel, blinding of outcome assessment, incomplete outcome data, selective reporting, and other bias. Then, according to the results of the table, it is divided into low risk of bias, unclear risk of bias and high risk of bias. In addition, we will resolve disagreements in the assessment through consensus or discussions with a third investigator.

\section{Data synthesis}

\section{Strategy for data synthesis}

The primary tool for data analysis will be Review Manager software (RevMan5.3). A random-effects model will be managed with data indicators from all of the studies for meta-analysis. We will evaluate and analyse the data on the overall included studies and summarise its 95\% CIs using a random-effects model. Differences will be considered statistically significant when the $p$ value is less than 0.05 . The funnel plot will be used to evaluate heterogeneity between overall included studies, such as differences in study types, risk of bias, publication bias, differences in measurement resolution and so on. ${ }^{34}$
Analysis of subgroups or subsets

Subgroup analysis will be carried out if there are sufficient data. Several subgroup analyses will be used to examine differences between the types of ET (eg, upper limbs, lower limbs and less commonly, the head, voice, tongue, face, trunk and others); age; the different dosages of propranolol; side effects of propranolol; the different therapies for ET; and study designs (eg, treatment groups vs no control group, RCTs vs non-RCTs). Among these variables, the dosage of propranolol and the types of ET are assumed to be the most important, as it remains unknown what dosage of propranolol is the most effective against different types of ET.

\section{DISCUSSION}

Treatments for ET can be mainly subdivided into three categories: medicine (propranolol, primidone, topiramate), surgical treatments (deep brain stimulation, gamma knife surgery, MRIgFUS (MRI-guided focused ultrasound)) and other therapies (botulinum toxin, lifestyle management). ${ }^{30}$ Thus far, there has not been a major breakthrough in ET treatments. Propranolol and primidone are generally the first treatment options in treating ET. Propranolol was proven to be effective against the treatment of ET in $1973 .{ }^{35}$ Furthermore, published controlled trials have shown that the average effective dose of propranolol is $185.2 \mathrm{mg} /$ day, and the daily dose range is $60-800 \mathrm{mg} /$ day ${ }^{36}{ }^{37} \mathrm{In}$ addition, there is insufficient evidence indicating that a dose over $320 \mathrm{mg}$ per day would provide any benefits. In the treatment of ET, an approximately $50 \%-70 \%$ response was observed. Compared with placebo, the average tremor can be reduced by approximately $50 \% .^{36} 38$

The primary objective of this systematic review will be to evaluate the efficacy and safety of propranolol in the treatment of ET. We will conduct a qualitative and quantitative analysis of the overall data included in each study, and we will hopefully find the optimal drug dose for the treatment of the ET subgroup. Furthermore, we will summarise as much as possible the role of propranolol in the treatment of ET, especially for axial tremors. The limitations of this systematic review are mainly due to the heterogeneity of the methodology, which may result in some results not being analysed.

\section{Twitter Hui Shen @shenhui}

Contributors ZZ, TW, MZ and WL designed the study. The draft agreement was drafted by ZZ, TW and MZ, and revised by all authors. MZ and WL designed the search strategy. MZ, WL and LH will perform the search. LC, LY, TZ and HS will be included in the study screening to extract data and assess the risk of bias in the included studies. ZZ and YP will dispute disagreements between reviewers. MZ, WL, SG and ZC will analyse and interpret the data. All authors agree to be responsible for all aspects of the work and have read and approved the final draft.

Funding This work was partially supported by a grant from National Natural Science Foundation of China (grant numbers 31260231 and 81860238); Major Research and Development Project of Hainan Province of China (grant number ZDYF2018233); General Research Projects of Educational and Teaching Reform in Colleges and Universities of Hainan Province of China (grant number HNJG201854); Scientific Research Projects of Health Department of Hainan Province of 
China (grant number 2018-10); Educational Research Projects of Hainan Medical University (grant number HYZ201705).

Competing interests None declared.

Patient consent for publication Not required.

Provenance and peer review Not commissioned; externally peer reviewed.

Open access This is an open access article distributed in accordance with the Creative Commons Attribution Non Commercial (CC BY-NC 4.0) license, which permits others to distribute, remix, adapt, build upon this work non-commercially, and license their derivative works on different terms, provided the original work is properly cited, appropriate credit is given, any changes made indicated, and the use is non-commercial. See: http://creativecommons.org/licenses/by-nc/4.0/.

\section{ORCID iDs}

Manyu Zhang http://orcid.org/0000-0002-0078-4429

Hui Shen http://orcid.org/0000-0002-6667-9744

\section{REFERENCES}

1 Benito-León J, Bermejo-Pareja F, Morales J-M, et al. Prevalence of essential tremor in three elderly populations of central Spain. Mov Disord 2003;18:389-94.

2 Louis ED, Ferreira JJ. How common is the most common adult movement disorder? Update on the worldwide prevalence of essential tremor. Mov Disord 2010;25:534-41.

3 Hopfner F, Deuschl G. Is essential tremor a single entity? Eur J Neurol 2018;25:71-82.

4 Louis ED. The roles of age and aging in essential tremor: an epidemiological perspective. Neuroepidemiology 2019;52:111-8.

5 Lee D, Gan S-R, Faust PL, et al. Climbing fiber-Purkinje cell synaptic pathology across essential tremor subtypes. Parkinsonism Relat Disord 2018;51:24-9.

6 Choe M, Cortés E, Vonsattel J-PG, et al. Purkinje cell loss in essential tremor: random sampling quantification and nearest neighbor analysis. Mov Disord. 2016;31:393-401.

7 Louis ED, Lee M, Babij R, et al. Reduced Purkinje cell dendritic arborization and loss of dendritic spines in essential tremor. Brain 2014;137:3142-8.

8 Kuo S-H, Erickson-Davis C, Gillman A, et al. Increased number of heterotopic Purkinje cells in essential tremor. J Neurol Neurosurg Psychiatry 2011;82:1038-40.

9 Haubenberger D, Hallett M. Essential tremor. N Engl J Med 2018;379:1802-10.

10 Juttukonda MR, Franco G, Englot DJ, et al. White matter differences between essential tremor and Parkinson disease. Neurology 2019;92:e30-9.

11 Gironell A. The GABA hypothesis in essential tremor: lights and shadows. Tremor Other Hyperkinet Mov 2014;4:254.

12 Espay AJ, Lang AE, Erro R, et al. Essential pitfalls in "essential" tremor. Mov Disord 2017;32:325-31.

13 Hsu YD, Chang MK, Sung SC, et al. Essential tremor: clinical, electromyographical and pharmacological studies in 146 Chinese patients. Zhonghua Yi Xue Za Zhi 1990;45:93-9.

14 Louis ED. Diagnosis and management of tremor. Continuum 2016;22:1143-58.

15 Chen W, Hopfner F, Szymczak S, et al. Topography of essential tremor. Parkinsonism Relat Disord 2017;40:58-63.
16 Louis ED. When do essential tremor patients develop head tremor? influences of age and duration and evidence of a biological clock. Neuroepidemiology 2013;41:110-5.

17 Albanese A, Dystonia SFD. Dystonia and tremor: the clinical syndromes with isolated tremor. Tremor Other Hyperkinet Mov 2016;6:319.

18 Bhatia KP, Bain P, Bajaj N, et al. Consensus statement on the classification of tremors. from the task force on tremor of the International Parkinson and movement disorder Society. Mov Disord 2018;33:75-87.

19 Witjas T, Carron R, Boutin E, et al. Essential tremor: update of therapeutic strategies (medical treatment and gamma knife thalamotomy). Rev Neurol 2016;172:408-15.

20 Hedera P, Cibulčík F, Davis TL. Pharmacotherapy of essential tremor. $J$ Cent Nerv Syst Dis 2013:5:43-55.

21 Louis ED. Essential tremor with head tremor: trait or state? Can J Neurol Sci 2016;43:443-4.

22 Rajalingam R, Breen DP, Lang AE, et al. Essential tremor plus is more common than essential tremor: insights from the reclassification of a cohort of patients with lower limb tremor. Parkinsonism Relat Disord 2018;56:109-10.

23 Patel A, Frucht SJ. Isolated vocal tremor as a focal phenotype of essential tremor: a retrospective case review. J Clin Mov Disord 2015;2:4.

24 Broersma M, van der Stouwe AMM, Buijink AWG, et al. Bilateral cerebellar activation in unilaterally challenged essential tremor. Neuroimage 2016;11:1-9.

25 Zakin E, Simpson D. Botulinum toxin in management of limb tremor. Toxins 2017;9:365.

26 Paparella G, Ferrazzano G, Cannavacciuolo A, et al. Differential effects of propranolol on head and upper limb tremor in patients with essential tremor and dystonia. J Neurol 2018;265:2695-703.

27 Guglielmino G, Moraes BT, Villanova LC, et al. Comparison of botulinum toxin and propranolol for essential and dystonic vocal tremors. Clinics 2018;73:e87.

28 Bain P, Brin M, Deuschl G, et al. Criteria for the diagnosis of essential tremor. Neurology 2000;54:S7.

29 Deuschl G, Bain P, Brin M. Consensus statement of the movement disorder Society on tremor. AD hoc scientific Committee. Mov Disord 1998;13:2-23.

30 Mittal SO, Lenka A, Jankovic J. Botulinum toxin for the treatment of tremor. Parkinsonism Relat Disord 2019;63:31-41.

31 Bruno E, Nicoletti A, Quattrocchi G, et al. Topiramate for essential tremor. Cochrane Database Syst Rev 2017;4:CD009683.

32 Moher D, Shamseer L, Clarke M, et al. Preferred reporting items for systematic review and meta-analysis protocols (PRISMA-P) 2015 statement. Syst Rev 2015;4:1.

33 Guyatt GH, Oxman AD, Vist GE, et al. GRADE: an emerging consensus on rating quality of evidence and strength of recommendations. BMJ 2008;336:924-6.

34 Egger M, Smith GD, Schneider M, et al. Bias in meta-analysis detected by a simple, graphical test. BMJ 1997;315:629-34.

35 Dupont E, Hansen HJ, Dalby MA. Treatment of benign essentia tremor with propranolol: a controlled clinical trial. Acta Neurol Scand 1973;49:75-84.

36 Koller WC. Dose-Response relationship of propranolol in the treatment of essential tremor. Arch Neurol 1986;43:42-3.

37 Cleeves L, Findley LJ. Propranolol and propranolol-LA in essential tremor: a double blind comparative study. J Neurol Neurosurg Psychiatry 1988;51:379-84.

38 Koller WC, Royse VL. Time course of a single oral dose of propranolol in essential tremor. Neurology 1985;35:1494-8. 